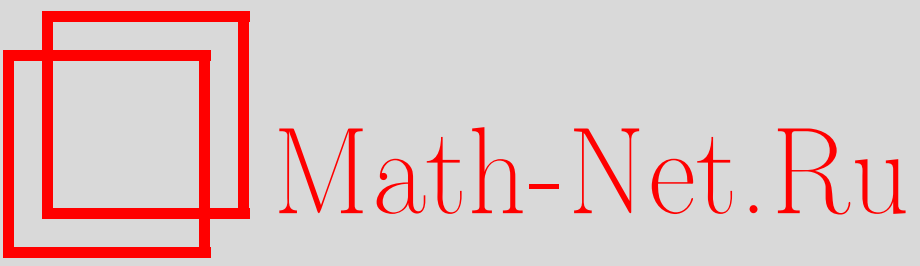

С. Е. Конштейн, Суперследы на алгебре наблюдаемых рациональной модели Калоджеро, основанной на классической системе корней, ТМФ, 1997, том 111, номер 2, 252-262

DOI: https://doi.org/10.4213/tmf1005

Использование Общероссийского математического портала Math-Net.Ru подразумевает, что вы прочитали и согласны с пользовательским соглашением

http: //www . mathnet.ru/rus/agreement

Параметры загрузки:

IP: 3.91 .87 .62

26 апреля 2023 г., 18:16:39 


\section{С.Е. Конштейн* \\ СУПЕРСЛЕДЫ НА АЛГЕБРЕ \\ НАБЛЮДАЕМЫХ РАЦИОНАЛЬНОЙ МОДЕЛИ КАЛОДЖЕРО, ОСНОВАННОЙ НА КЛАССИЧЕСКОЙ СИСТЕМЕ КОРНЕЙ}

\footnotetext{
Определен полный набор суперследов на алгебрах $H_{W(\mathbf{R})}(\nu)$ наблюдаемых рациональных моделей Калоджеро с гармоническим потенциалом, основанных на системах корней $\mathbf{R}$ типов $B_{N}, C_{N}$ и $D_{N}$. Эти результаты обобщают результаты, известные для случая $A_{N-1}$. Показано, что $H_{W(\mathbf{R})}(\nu)$ имеет $q(\mathbf{R})$ независимых суперследов, где $q\left(B_{N}\right)=q\left(C_{N}\right)$ - число разбиений числа $N$ в сумму целых положительных чисел, а $q\left(D_{N}\right)$ есть число разбиений числа $N$ в сумму целых положительных чисел с четным числом четных слагаемых.
}

\section{1. ВВЕДЕНИЕ}

В этой работе мы продолжаем исследование некоторых свойств ассоциативных алгебр, которые, как было показано в $[1,2]$, лежат в основе интегрируемости рациональной модели Калоджеро [3]. Мы распространили результаты, полученные в [4], на ассоциативные алгебры наблюдаемых рациональной модели Калоджеро, связанной с системами корней [5] классических алгебр Ли $B_{N}, C_{N}$ и $D_{N}$, показав, что и на этих супералгебрах существуют нетривиальные суперследы.

Пусть $H_{G}(\nu)$ - ассоциативная алгебра полиномов, порожденная $N$ парами (деформированных) осцилляторов Гейзенберга-Вейля $a_{i}^{\alpha}, \alpha=1,2, i=1, \ldots, N$, образуюших базис в паре $N$-мерных пространств $a_{i}^{\alpha} \in \mathcal{H}_{N}^{\alpha}$, и элементами некоторой конечной группы $G$, удовлетворяюшая следуюшим условиям:

1. На $H_{G}(\nu)$ задана четность $\pi: \pi\left(a_{i}^{\alpha}\right)=1, \pi(g)=0 \forall g \in G$.

2. $g a_{i}^{\alpha}=\sum_{j} T_{i j}\left(g^{-1}\right) a_{j}^{\alpha} g \forall g \in G$ и для всех $\alpha=1,2, i=1, \ldots, N, a_{i}^{\alpha} \in \mathcal{H}_{N}^{\alpha}$, где ортогональные матрицы $T_{i j}(g)$ реализуют $N$-мерное представление группы $G$.

3. $\left[a_{i}^{\alpha}, a_{j}^{\beta}\right]=\epsilon^{\alpha \beta} A_{i j}$, где $\epsilon^{\alpha \beta}$ - антисимметричный тензор и компоненты $A_{i j}=$ $A_{j i} \in \mathfrak{G}(\mathfrak{G}-$ групповая алгебра группы $G)$ зависят от одного или более параметров $\nu$.

Чтобы сформулировать следуюшее условие, введем подпространства $\mathcal{E}^{\alpha}(g) \subset \mathcal{H}_{N}^{\alpha}$, равные

$$
\mathcal{E}^{\alpha}(g)=\left\{h \in \mathcal{H}_{N}^{\alpha}: g h=-h g\right\}
$$

\footnotetext{
* Физический институт РАН им. П. Н. Лебедева, отдел теоретической физики им. И. Е. Тамма, Москва, Россия
} 
и градуировку $E$ на $\mathfrak{G}$

$$
E(g)=\operatorname{dim} \mathcal{E}^{\alpha}(g) .
$$

4. $E\left(\mathcal{P}\left(\left[h_{0}, h_{1}\right]\right) g\right)=E(g)-1 \forall g \in G, \forall h_{\alpha} \in \mathcal{E}^{\alpha}(g)$. Через $\mathcal{P}(g)$ здесь обозначен проектор $\mathfrak{G} \rightarrow \mathfrak{G}$, определенный как $\mathcal{P}\left(\sum_{i} \alpha_{i} g_{i}\right)=\sum_{i: g_{i} \neq 1} \alpha_{i} g_{i}$ для любых $g_{i} \in G$ и произвольных констант $\alpha_{i}$.

Суперслед определяется как линейная комплекснозначная функция $\operatorname{str}(\cdot)$ на алгебре $H_{G}(\nu)$ такая, что

$$
\operatorname{str}(f g)=(-1)^{\pi(f) \pi(g)} \operatorname{str}(g f) \quad \forall f, g \in H_{G}(\nu) .
$$

В [4] на примере $G=S_{N}$ была доказана следуюшая теорема.

ТЕОРема 1. Если супералгебра $H_{G}(\nu)$ удовлетворяет условиям 1-4, то количество независимых нетривиальных суперследов на $H_{G}(\nu)$ равно количеству независимых суперследов на алгебре $\mathfrak{G}$, удовлетворяющих уравнению

$$
\operatorname{str}\left(\left[h_{0}, h_{1}\right] g\right)=0 \quad \forall h_{\alpha} \in \mathcal{E}^{\alpha}(g), \quad \forall g \in G .
$$

В настоящей работе эта теорема применяется к случаю, когда $G$ является группой Вейля $W(\mathbf{R})$ системы корней $\mathbf{R}$ классической алгебры Ли типа $A_{N-1}, B_{N}, C_{N}$ или $D_{N}$. Мы будем рассматривать три (т.к. $\left.W\left(B_{N}\right)=W\left(C_{N}\right)\right)$ различных случая одновременно. Заметим, что алгебра $S H_{N}(\nu)$, рассматривавшаяся в $[6,4]$, совпадает в наших обозначениях с $H_{W\left(A_{N-1}\right)}(\nu)=H_{S_{N}}(\nu)$.

Рассмотрим 3-параметрическую деформацию $H_{G}(\nu)$ ассоциативной алгебры Гейзенберга-Вейля $N$ пар осцилляторов. Эта деформация порождена $N$ парами порождающих элементов $a_{i}^{\alpha}, \alpha=1,2$, и отражениями $K_{i j}$ и $R_{i}, i, j=1, \ldots, N$, удовлетворяющими следующим соотношениям ${ }^{1)}$ :

$$
\begin{gathered}
K_{i j}=K_{j i}, \quad K_{i j} K_{i j}=1, \quad K_{i j} K_{j l}=K_{j l} K_{l i}=K_{l i} K_{i j} \quad \text { для } i \neq j \neq l \neq i, \\
K_{i j} K_{k l}=K_{k l} K_{i j}, \quad \text { если } i, j, k, l \text { попарно различны, } \\
R_{i} R_{j}=R_{j} R_{i} \forall i, j, \quad R_{i} R_{i}=1 \quad \forall i, \\
K_{i j} R_{j}=R_{i} K_{i j}, \quad K_{i j} R_{k}=R_{k} K_{i j} \text { для } i \neq j \neq k \neq i, \\
R_{i} a_{i}^{\alpha}=-a_{i}^{\alpha} R_{i}, \quad R_{i} a_{j}^{\alpha}=a_{j}^{\alpha} R_{i} \text { для } i \neq j, \\
K_{i j} a_{j}^{\alpha}=a_{i}^{\alpha} K_{i j}, \quad K_{i j} a_{k}^{\alpha}=a_{k}^{\alpha} K_{i j} \text { для } i \neq j \neq k \neq i, \\
{\left[a_{i}^{\alpha}, a_{j}^{\beta}\right]=\epsilon^{\alpha \beta} A_{i j},}
\end{gathered}
$$

где $\epsilon^{\alpha \beta}=-\epsilon^{\beta \alpha}, \epsilon^{01}=1$ и

$$
\begin{aligned}
& A_{i j}=\delta_{i j}+\nu_{0} \tilde{A}_{i j}^{0}+\nu_{1} \tilde{A}_{i j}^{1}+\nu_{2} \tilde{A}_{i j}^{2}, \\
& \tilde{A}_{i j}^{0}=\delta_{i j} \sum_{l=1, l \neq i}^{N} K_{i l}-\delta_{i \neq j} K_{i j}, \\
& \tilde{A}_{i j}^{1}=\delta_{i j} R_{i}, \\
& \tilde{A}_{i j}^{2}=\delta_{i j} \sum_{l \neq i} K_{i l} R_{i} R_{l}+\delta_{i \neq j} K_{i j} R_{i} R_{j} .
\end{aligned}
$$

\footnotetext{
${ }^{1)} \mathrm{B}$ настоящей статье одинаковые латинские индексы $i, j, k, \ldots$ не обозначают суммирование.
} 
Коммутационные соотношения, определенные таким образом, являются непротиворечивыми, если вьполнено одно из следующих условий:

А) $\nu_{1}=\nu_{2}=0$, и образующих $R_{i}$ в алгебре нет,

$\mathrm{B}, \mathrm{C}) \nu_{0}=\nu_{2}$

D) $\nu_{0}=\nu_{2}, \nu_{1}=0$, и каждый моном в $H_{G}(\nu)$ содержит четное число отражений $R_{i}$.

Отражения $K_{i j}$ и $R_{i}$ определяют группу Вейля системы корней $A_{N-1}, B_{N}, C_{N}$ или $D_{N}$ соответственно этим случаям, а для операторов $a_{i}^{\alpha}$ имеется представление

$$
a_{i}^{\alpha}=\frac{1}{\sqrt{2}}\left(x_{i}+(-1)^{\alpha} D_{i}(x)\right)
$$

где $D_{i}(x)$ - дифференциально-разностные операторы Данкла [7], связанные с соответствующей системой корней

$$
D_{i}=\frac{\partial}{\partial x_{i}}+\nu_{1} \frac{1}{x_{i}}\left(1-R_{i}\right)+\sum_{l \neq i}^{N}\left(\nu_{0} \frac{1}{x_{i}-x_{l}}\left(1-K_{i l}\right)+\nu_{2} \frac{1}{x_{i}+x_{l}}\left(1-K_{i l} R_{i} R_{l}\right)\right)
$$

и удовлетворяюшие соотношениям

$$
\left[D_{i}, D_{j}\right]=0 \quad \forall i, j
$$

для значений параметров $\nu$, перечисленных выше ${ }^{2)}$.

Гамильтониан рациональной модели Калоджеро, связанной с соответствующей системой корней, идентифицируется с дифференциальным оператором второго порядка $H=\frac{1}{2} \sum_{i=1}^{N}\left\{a_{i}^{0}, a_{i}^{1}\right\}$. При этом операторы $a_{i}^{1}$, действуя на фоковский вакуум $|0\rangle$ такой, что $a^{0}|0\rangle=0$, дают волновые функции этой модели.

Знание суперследов на $H_{W(\mathbf{R})}(\nu)$ полезно в различных отношениях. Они определяют полилинейные инвариантные формы

$$
\operatorname{str}\left(a_{1} a_{2} \ldots a_{n}\right)
$$

что позволяет, например, строить лагранжианы при работе с динамическими теориями, основанными на этих алгебрах. Кроме того, т.к. нуль-векторы некоторой инвариантной билинейной формы образуют двусторонний идеал алгебры, то знание суперследов дает мощный инструмент для исследования таких идеалов (как, например, в случае $H_{W\left(A_{1}\right)}(\nu)$, соответствуюшем обычной двухчастичной модели Калоджеро при полуцелых $\nu$ [8]).

Важной мотивацией для анализа суперследов на $H_{W(\mathbf{R})}(\nu)$ является то, что они имеют тесную связь с представлениями этой алгебры, а значит, и с анализом волновых функций модели Калоджеро. Например, имея представление $H_{W(\mathbf{R})}(\nu)$, можно предположить, что оно индуцирует некоторьй суперслед этой алгебры как (регуляризованньй тем или иным способом) суперслед (бесконечной) матрицы представления. Когда соответствующая билинейная форма при некоторых значениях параметров $\nu$ вырождается, это может означать, что представление становится приводимым.

\footnotetext{
2) В формуле (13) отражения действуют на функции от $x_{i}$ по правилу $K_{i j} f\left(x_{i}, x_{j}\right)=f\left(x_{i}, x_{j}\right)$, $R_{i} f\left(x_{i}\right)=f\left(-x_{i}\right)$.
} 
Почти для всех рассмотренных в настоящей работе алгебр ситуация является довольно интересной, т.к. почти все они имеют более одного суперследа. Для конечномерных алгебр этого достаточно, чтобы они имели двусторонние идеалы, однако для рассматриваемых бесконечномерных алгебр сушествование идеалов пока является открытой проблемой.

Ниже в разделе 2 доказана лемма, обеспечиваюшая сушествование суперследов на $H_{W(\mathbf{R})}(\nu)$, а в разделе 3 при помощи теоремы, доказанной в приложении, подсчитано их количество.

\section{2. ОДНОМЕРНЫЕ ПРЕДСТАВЛЕНИЯ ЭЛЕМЕНТОВ ГРУППЫ ВЕЙЛЯ КЛАССИЧЕСКОЙ СИСТЕМЫ КОРНЕЙ}

В этом разделе доказывается, что $H_{G}(\nu)$ обладает свойством 4 , описанным вьше, если группа $G$ есть группа Вейля классической системы корней, порожденная отражениями $K_{i j}$ и $R_{i}$.

Всякий элемент $g \in G$ при $G=W\left(A_{N-1}\right), G=W\left(B_{N}\right), G=W\left(C_{N}\right)$ и $G=W\left(D_{N}\right)$ может быть представлен в виде $g=\sigma \prod_{i \in M_{q}} R_{i}$, где $\sigma \in S_{N}$ - подстановка, а $M_{g}$ некоторое подмножество индексов $1, \ldots, N$. Хорошо известно, что всякая подстановка $\sigma \in S_{N}$ разбивает множество индексов $1, \ldots, N$ на подмножества $C_{1}, \ldots, C_{t_{\sigma}}$, на которых она действует транзитивно циклическими подстановками $c_{1}, \ldots, c_{t_{\sigma}}$, соответственно. При этом $\sigma=\prod_{\mathfrak{m}=1}^{t_{\sigma}} c_{\mathfrak{m}}$.

Введем элементы

$$
r_{\mathfrak{m}}=\prod_{i \in M_{g} \cap C_{\mathfrak{m}}} R_{i}
$$

Тогда $g$ может быть представлен в виде

$$
g=\prod_{\mathfrak{m}=1}^{t_{\sigma}} \hat{c}_{\mathfrak{m}}, \quad \text { где } \quad \hat{c}_{\mathfrak{m}}=c_{\mathfrak{m}} r_{\mathfrak{m}} .
$$

Эти элементы $\hat{c}_{\mathfrak{m}}$ далее будем называть циклами.

Каждому элементу $g \in G$ поставим в соответствие вместо базисных элементов $\left\{a_{i}^{\alpha}\right\}$ новое множество базисных элементов $\mathfrak{B}_{g}=\left\{b^{I}\right\}$ следуюшим образом. Для каждого цикла $\hat{c}_{\mathfrak{m}}$ в разложении $(16)(\mathfrak{m}=1, \ldots, t)$ зафиксируем какой-нибудь индекс $l_{\mathfrak{m}}$, принадлежаший подмножеству $C_{\mathfrak{m}}$, ассоциированному с циклом $\hat{c}_{\mathfrak{m}}$. Введем базисные элементы $b_{\mathfrak{m} j}^{\alpha}, j=1, \ldots,\left|\widehat{C}_{\mathfrak{m}}\right|$, реализующие 1 -мерные представления коммутативной циклической группы, порожденной $\hat{c}_{\mathfrak{m}}$, в виде

$$
b_{\mathfrak{m} j}^{\alpha}=\frac{1}{\sqrt{\left|C_{\mathfrak{m}}\right|}} \sum_{k=1}^{\left|C_{\mathfrak{m}}\right|}\left(\lambda_{\mathfrak{m}}\right)^{j k} \hat{c}_{\mathfrak{m}}^{-k} a_{l_{\mathfrak{m}}}^{\alpha} \hat{c}_{\mathfrak{m}}^{k}
$$

где

$$
\lambda_{\mathfrak{m}}=\exp \left(\frac{1+\left(\left|\hat{c}_{\mathfrak{m}}\right|+\left|C_{\mathfrak{m}}\right|\right) \mid \bmod 2}{\left|C_{\mathfrak{m}}\right|} \pi i\right)
$$


Здесь через $\left|C_{\mathfrak{m}}\right|$ обозначено количество элементов в подмножестве $C_{\mathfrak{m}}$, а через $\left|\hat{c}_{\mathfrak{m}}\right|-$ длина цикла $\hat{c}_{\mathfrak{m}}$. Длина $|g|$ элемента $g \in G$ определяется как минимальное количество отражений, произведение которых равно $g^{3)}$.

Из определения (17) следует, что

$$
\begin{aligned}
& \hat{c}_{\mathfrak{m}} b_{\mathfrak{m} j}^{\alpha}=\left(\lambda_{\mathfrak{m}}\right)^{j} b_{\mathfrak{m} j}^{\alpha} \hat{c}_{\mathfrak{m}}, \\
& \hat{c}_{\mathfrak{m}} b_{\mathfrak{n} j}^{\alpha}=b_{\mathfrak{n} j}^{\alpha} \hat{c}_{\mathfrak{m}} \quad \text { для } \quad \mathfrak{n} \neq \mathfrak{m}
\end{aligned}
$$

и, следовательно,

$$
g b_{\mathfrak{m} j}^{\alpha}=\left(\lambda_{\mathfrak{m}}\right)^{j} b_{\mathfrak{m} j}^{\alpha} g .
$$

Теперь легко получить, что для всякого цикла $\hat{c}_{\mathfrak{m}}$ с четной длиной матрица $T_{i j}\left(\hat{c}_{\mathfrak{m}}\right)$ не имеет собственных значений, равных -1 , а для циклов с нечетной длиной сушествует ровно одно собственное значение, равное -1 . Вследствие этого определенная ранее функция $E(g)$ есть не что иное, как число нечетных циклов в разложении (16) элемента $g$.

В дальнейшем вместо обозначения $b_{\mathfrak{m} j}^{\alpha}$ мы будем использовать обозначение $b^{I}$, в котором индекс $I$ несет всю информацию об индексе $\alpha$, индексе $\mathfrak{m}$, нумерующем циклы в (16), и индексе $j$, нумеруюшем различные элементы $b_{\mathfrak{m} j}^{\alpha}$, связанные с циклом $\hat{c}_{\mathfrak{m}}$, т.е. $I$ $(I=1, \ldots, 2 N)$ перенумеровывает все возможные тройки $\{\alpha, \mathfrak{m}, j\}$. Будем обозначать индекс $\alpha$, цикл, подмножество индексов и собственное значение в (19), отвечающее некоторому индексу $I$, как $\alpha(I), c(I), C(I)$ и $\lambda_{I}=\left(\lambda_{\mathfrak{m}}\right)^{j}$, соответственно. Запись $g(I)=g_{0}$ означает, что $b^{I} \in \mathfrak{B}_{g_{0}}$. Базис $\mathfrak{B}_{1}$ является исходным базисом производяших элементов $a_{i}^{\alpha}$ (здесь $\mathbf{1} \in G$ - единичный элемент алгебры).

Пусть $\mathfrak{M}(g)$ - матриша, отображаюшая $\mathfrak{B}_{1} \rightarrow \mathfrak{B}_{g}$ в соответствии с (17),

$$
b^{I}=\sum_{i, \alpha} \mathfrak{M}_{i \alpha}^{I}(g) a_{i}^{\alpha}
$$

Очевидно, что она обратима. В матричных обозначениях (21) может быть переписано в виде

$$
g b^{I} g^{-1}=\sum_{J=1}^{2 N} \Lambda_{J}^{I}(g) b^{J} \quad \forall b^{I} \in \mathfrak{B}_{g}
$$

где $\Lambda_{I}^{J}(g)=\delta_{I}^{J} \lambda_{I}$

Коммутационные соотношения для образуюших элементов $b^{I}$ следуют из (10) и (11):

$$
\left[b^{I}, b^{J}\right]=\mathcal{C}^{I J}+\epsilon^{\alpha(I) \alpha(J)} B^{I J},
$$

где $g(I)=g(J)$,

$$
\mathcal{C}^{I J}=\epsilon^{\alpha(I) \alpha(J)} \delta_{c(I) c(J)} \delta_{\lambda_{I} \lambda_{J}^{-1}}
$$

и

$$
B^{I J}=\sum_{i, j, \alpha, \beta} \mathfrak{M}_{i \alpha}^{I}(\sigma) \mathfrak{M}_{i \beta}^{J}(\sigma) A_{i j} \epsilon^{\alpha \beta} \epsilon^{\alpha(I) \alpha(J)}, \quad \epsilon^{\alpha(I) \alpha(J)} B^{I J}=\mathcal{P}\left(\left[b^{I}, b^{J}\right]\right) .
$$

\footnotetext{
3) В [4] было использовано определение длины, на единицу отличающееся от этого.
} 
Индексы $I, J$ поднимаются и опускаются при помощи симплектической формы $\mathcal{C}^{I J}$ :

$$
b^{I}=\sum_{J} \mathcal{C}^{I J} b_{J}, \quad b_{I}=\sum_{J} b^{J} \mathcal{C}_{J I} ; \quad \sum_{M} \mathcal{C}_{I M} \mathcal{C}^{M J}=-\delta_{I}^{J}
$$

Заметим, что элементы $b^{I}$ нормированы в (17) таким образом, что $\nu$-независимая часть в (24) имеет вид (25).

Теперь мы можем при помощи теоремы 1 доказать существование суперследов в случаях $B_{N}, C_{N}$ и $D_{N}$. Для этого достаточно доказать следующую лемму (свойство 4$)$.

Лемма 1. Eсли $\lambda_{I}=\lambda_{J}=-1 u g(I)=g(J)=g$, mo $E\left(B^{I J} g\right)=E(g)-1$.

Доказательство основано на следуюших простых фактах теории симметрических групп.

ПРЕДЛОЖЕНИЕ 1. Пусть $c_{1}$ и $c_{2}$ - два различных иикла в разложсении некоторой подстановки из $S_{N}$. Пусть индексь $i_{1} u i_{2}$ принадлежсат подмножествам, ассоциированным с ииклами $c_{1}$ и $c_{2}$, соответственно. Тогда подстановка $c=K_{i_{1} i_{2}} c_{1} c_{2}$ есть иикл длиньи $|c|=\left|c_{1}\right|+\left|c_{2}\right|+1$.

ПРЕДЛОЖЕНИЕ 2. Пусть $c \in S_{N}-$ ииклическая подстановка и пусть $i \neq j-$ два индекса такие, что $c^{k}(i)=j$, где $k$ - некоторое положительное целое число, $k<|c|$. Тогда $K_{i j} c=c_{1} c_{2}$, где $c_{1,2}$ - несовпадающие коммутирующие иикль такие, что $\left|c_{1}\right|=k-1 u\left|c_{2}\right|=|c|-k$.

Для доказательства леммы 1 рассмотрим сначала случай $c(I)=c(J)$. Для определенности выберем нечетный цикл

$$
c(I)=c(J)=K_{12} K_{23} \ldots K_{(p-1) p} R_{i_{1}} R_{i_{2}} \ldots R_{i_{l}}
$$

(с $1 \leq i_{1}<i_{2}<\cdots<i_{l} \leq p$ и нечетным $p-1+l$ ) и

$$
b^{P}=\frac{1}{\sqrt{p}} \sum_{j=1}^{p}(-1)^{j}(-1)^{\Delta(j)} a_{j}^{\alpha(P)}, \quad P=I, J,
$$

где

$$
\Delta(j)=\sum_{m \geq j} \sum_{n=1}^{l} \delta_{m i_{n}}
$$

Тогда $B^{I J}$ может быть записано в следуюшем виде:

$$
\begin{aligned}
p B^{I J}= & \sum_{i=1, j=1, i \neq j}^{p}\left(\nu_{0}\left(1-(-1)^{i+j+\Delta(i)+\Delta(j)}\right) K_{i j}+\right. \\
& \left.+\nu_{2}\left(1+(-1)^{i+j+\Delta(i)+\Delta(j)}\right) K_{i j} R_{i} R_{j}\right)+ \\
& +\nu_{1} \sum_{i=1}^{p} R_{i}+\sum_{i=1}^{p} \sum_{j=p+1}^{N}\left(\nu_{0} K_{i j}+\nu_{2} K_{i j} R_{i} R_{j}\right) .
\end{aligned}
$$

При помощи предложений 1 и 2 легко убедиться, что:

4 Теоретическая и математическая физика, т. 111, № 2, 1997 г. 
1) $K_{i j} c(I)$ разлагается в произведение двух четных циклов, если $1 \leq i<j \leq p$ и $i+j+\Delta(i)+\Delta(j)$ нечетно;

2) $K_{i j} R_{i} R_{j} c(I)$ разлагается в произведение двух четных циклов, если $1 \leq i<j \leq p$ и $i+j+\Delta(i)+\Delta(j)$ четно;

3) $R_{i} c(I)$ является четным циклом, если $1 \leq i \leq p$, т.к. $c(I)$ - нечетный цикл;

4) $K_{i j} c(I) c(K)$ есть цикл с той же четностью, что и $c(K)$, если $c(K) \neq c(I)$ - некоторый цикл в разложении (16) элемента $g, i \in C(I)$ и $j \in C(K)$;

5) $K_{i j} R_{i} R_{j} c(I) c(K)$ есть цикл с той же четностью, что и $c(K)$, если $c(K) \neq c(I)-$ некоторьй цикл в разложении (16) элемента $g, i \in C(I)$ и $j \in C(K)$.

Случай $c(I) \neq c(J)$ сводится к подслучаям 4 и 5 , что завершает доказательство леммы 1.

\section{3. СУПЕРСЛЕДЫ НА $\mathfrak{G}$, ВАКУУМНЫЕ УСЛОВИЯ И ЧИСЛО СУПЕРСЛЕДОВ НА $H_{W(\mathbf{R})}(\nu)$}

Вследствие $G$-инвариантности определение суперследа на $\mathfrak{G}$ является определением центральной функции на $\mathfrak{G}$, т.е. функции на классах сопряженности $G$, и поэтому число суперследов на $\mathfrak{G}$ равно числу классов сопряженности в $G$.

Так как $\mathfrak{G} \subset H_{G}(\nu)$, то из (3) и определяющих соотношений $(5)-(11)$ алгебры $H_{G}(\nu)$ следуют дополнительные ограничения на эти функции. Действительно, рассмотрим элементы $b^{I}$ такие, что $\lambda_{I}=-1$. Тогда из (3) и (21) следует, что $\operatorname{str}\left(b^{I} b^{J} g\right)=$ $-\operatorname{str}\left(b^{J} g b^{I}\right)=\operatorname{str}\left(b^{J} b^{I} g\right)$, и, следовательно, $\operatorname{str}\left(\left[b^{I}, b^{J}\right] g\right)=0$, что вследствие $(24)$ и $(25)$ эквивалентно

$$
\delta_{c(I) c(J)} \delta_{-1 \lambda_{J}} \operatorname{str}(g)=-\operatorname{str}\left(B^{I J} g\right) .
$$

Так как эти условия дают ограничения на суперследы полиномов степени 0 по $a_{i}^{\alpha}$, мы назвали их в [4] вакуумными условиями (Ground Level Conditions). В силу леммы 2, доказанной в приложении, уравнения (32) удовлетворяются тождественно для любого суперследа на $\mathfrak{G}$, если $\delta_{c(I) c(J)} \delta_{-1 \lambda_{J}}=0$, поэтому они выражают суперслед элементов $g$ с $E(g)=e$ через суперслед элементов $B^{I J} g$ с $E\left(B^{I J} g\right)=e-1$ :

$$
\operatorname{str}(g)=-\operatorname{str}\left(B^{I} g\right)
$$

где через $B^{I}$ обозначена величина $B^{I J}$ такая, что $c_{J}=c_{I}, \lambda_{I}=\lambda_{J}=-1, \alpha(I)+$ $\alpha(J)=1$. Таким образом, число решений этой системы $Q(G)$ не превосходит $\mathcal{O}(G)-$ количества классов сопряженности элементов без нечетных циклов.

В приложении доказана следующая теорема.

Теорема 2. Если $G=W(\mathbf{R}) u \mathbf{R}=A_{N-1}, B_{N}, C_{N}$ uлu $D_{N}, \operatorname{mo} Q(G)=\mathcal{O}(G)$.

Чтобы вычислить величину $\mathcal{O}(G)$, рассмотрим некоторый четньй цикл

$$
c=K_{12} K_{23} \ldots K_{(p-1) p} R_{i_{1}} R_{i_{2}} \ldots R_{i_{l}} \quad(p-1+l \text { четно })
$$

и цепочку преобразований подобия допустимых во всех случаях ${ }^{4)} \mathbf{R}=B_{N}, C_{N}, D_{N}$ :

$$
\begin{aligned}
c \rightarrow R_{i_{l}} R_{i_{l}-1} c R_{i_{l}} R_{i_{l}-1} & =K_{12} K_{23} \ldots K_{(p-1) p} R_{i_{1}} R_{i_{2}} \ldots R_{i_{l}-2} \rightarrow \cdots \rightarrow= \\
& =K_{12} K_{23} \ldots K_{(p-1) p}\left(R_{1}\right)^{l_{1}}\left(R_{2}\right)^{l-l_{1}} .
\end{aligned}
$$

\footnotetext{
${ }^{4)}$ Отражения $R_{i}$ отсутствуют в случае $\mathbf{R}=A_{N-1}$.
} 
Здесь $l_{1}$ - число нечетных индексов $i_{k}$ в $(34)$. Если $p$ четно, то либо $l_{1}$, либо $l-l_{1}$ нечетно и элемент (34) подобен $K_{12} K_{23} \ldots K_{(p-1) p} R_{1}$. Если $p$ нечетно, то элемент $(34)$ подобен либо $K_{12} K_{23} \ldots K_{(p-1) p}$, либо $K_{12} K_{23} \ldots K_{(p-1) p} R_{1} R_{2}$. Последнее выражение подобно $K_{12} K_{23} \ldots K_{(p-1) p} R_{1} R_{p}$, а это, в свою очередь, подобно $K_{12} K_{23} \ldots K_{(p-1) p}$, т.к. $p$ нечетно.

Это рассмотрение показывает, что класс сопряженности четного цикла $c(I)$ определяется двумя зависимыми величинами: числом индексов $p(I)=|C(I)|$, на которые действует $c(I)$, и четностью $\epsilon(I)=(p(I)+1) \bmod 2$ числа отражений $R$ в $c(I)$.

Таким образом, класс сопряженности с $E \stackrel{\text { mod }}{=}$ полностью характеризуется набором неотрицательных целых чисел $n_{1}, n_{3}, \ldots ; m_{2}, m_{4}, \ldots$, где $n_{i}-$ это число циклов $c(I)$ с $p(I)=i$ и $\epsilon(I)=0$, а $m_{i}$ - число циклов $c(I)$ с $p(I)=i$ и $\epsilon(I)=1$.

Числа $n_{i}$ и $m_{i}$ должны удовлетворять следуюшим условиям:

$$
\begin{aligned}
& \text { для случая "А" } m_{i}=0 \forall i, \sum_{i} i n_{i}=N \text {; } \\
& \text { для случаев "B", "C" } \sum_{i} i\left(n_{i}+m_{i}\right)=N ; \\
& \text { для случая "D" } \sum_{i} i\left(n_{i}+m_{i}\right)=N,\left(\sum_{i} m_{i}\right)_{\bmod 2}=0 .
\end{aligned}
$$

Таким образом, число суперследов равно числу разбиений числа $N$ в сумму нечетных положительных целых для случая $A_{N-1}$, числу разбиений числа $N$ в сумму целых для случаев $B_{N}$ и $C_{N}$ и числу разбиений числа $N$ в сумму положительных целых с четным числом четных слагаемых для $D_{N}$.

Автор благодарен М. А. Васильеву за полезные обсуждения. Настоящая работа была частично поддержана Российским фондом фундаментальных исследований, грант 96-01-01144, и INTAS, грант 93-0633.

\section{Доказательство теоремы 2}

ПРИЛОЖКНИЕ

Сначала докажем следующую лемму:

Лемма 2. Уравнения $\operatorname{str}\left(\left[b^{I}, b^{J}\right] g\right)=0 \quad c \quad b^{I}, b^{J} \in \mathfrak{B}_{g}, \quad \lambda_{I}=-1$ удовлетворяются тождественно для всякого суперследа $\operatorname{str}(\cdot)$ на $\mathfrak{G}$, если $c(I) \neq c(J)$ или $c(I)=c(J) u \lambda_{J} \neq-1$.

Действительно, вследствие $G$-инвариантности можно получить следующие тождества:

$$
\begin{aligned}
\operatorname{str}\left(\left[b^{I}, b^{J}\right] g\right) & =\operatorname{str}\left(c(I)\left[b^{I}, b^{J}\right] g(c(I))^{-1}\right)= \\
& =\operatorname{str}\left(\left[c(I) b^{I}(c(I))^{-1}, c(I) b^{J}(c(I))^{-1}\right] c(I) g(c(I))^{-1}\right)=\operatorname{str}\left(\left[-b^{I}, b^{J}\right] g\right)
\end{aligned}
$$

для случая $c(I) \neq c(J)$ и

$$
\operatorname{str}\left(\left[b^{I}, b^{J}\right] g\right)=\operatorname{str}\left(c(I)\left[b^{I}, b^{J}\right] g(c(I))^{-1}\right)=-\lambda_{J} \operatorname{str}\left(\left[b^{I}, b^{J}\right] g\right)
$$

для случая $c(I)=c(J)$.

В результате для доказательства теоремы 2 нужно рассматривать только случай $c(I)=c(J), \lambda_{I}=\lambda_{J}=-1$ и $\alpha(I)=1-\alpha(J)$.

Индукцией по числу нечетных циклов $e=E(g)$ покажем, что для $g$ с $E(g)=e \geq 1$ есть только одно независимое уравнение на $\operatorname{str}(g)$ при условии, что все уравнения (33) с 
$E(g)=e^{\prime}<e$ разрешены. Первый шаг индукции состоит в наблюдении, что для случая $E(g)=0$ уравнений нет.

Рассмотрим теперь вариант, когда есть два уравнения (33) на $\operatorname{str}(g)$ для некоторого $g$. Такое возможно только тогда, когда $g=c_{1} c_{2} g^{\prime}$, где $c_{1}$ и $c_{2}-$ нечетные циклы в разложении $g$ такие, что $c_{1}$ не подобен $c_{2}$. Заметим, что $E\left(g^{\prime}\right)=E(g)-2=e-2$, $E\left(c_{1} g^{\prime}\right)=E\left(c_{2} g^{\prime}\right)=e-1$.

Без потери обшности положим

$$
c_{1}=K_{12} K_{23} \ldots K_{(p-1) p} R_{i_{1}} R_{i_{2}} \ldots R_{i_{k}}
$$

с $1 \leq i_{1}<i_{2}<\cdots<i_{k} \leq p$ и нечетным $p-1+k$ и

$$
c_{2}=K_{(p+1)(p+2)} \ldots K_{(p+q-1)(p+q)} R_{i_{k+1}} R_{i_{k+2}} \ldots R_{i_{k+l}}
$$

с $p+1 \leq i_{k+1}<i_{k+2}<\cdots<i_{k+l} \leq p+q$ и нечетным $q-1+l$ и введем соответствуюшие образуюшие элементы

$$
\begin{aligned}
& b_{1}^{\alpha}=\frac{1}{\sqrt{p}} \sum_{s=1}^{p}(-1)^{s}(-1)^{\Delta(s)} a_{s}^{\alpha}, \\
& b_{2}^{\alpha}=\frac{1}{\sqrt{q}} \sum_{s=p+1}^{p+q}(-1)^{s}(-1)^{\Delta(s)} a_{s}^{\alpha},
\end{aligned}
$$

где

$$
\Delta(s)=\sum_{u=s}^{p+q} \delta(u), \quad \delta(u)=\sum_{v=1}^{k+l} \delta_{u i_{v}}
$$

с собственными значениями $\lambda_{1}=\lambda_{2}=-1$.

Заметим, что $c_{+}=K_{1(p+1)} c_{1} c_{2}$ есть цикл такой, что

$$
c_{+} b_{+}^{\alpha}=-b_{+}^{\alpha} c_{+}, \quad \text { где } \quad b_{+}^{\alpha}=\frac{\sqrt{p} b_{1}^{\alpha}+\sqrt{q} b_{2}^{\alpha}}{\sqrt{p+q}},
$$

a $c_{-}=K_{1 p+1} R_{1} R_{p+1} c_{1} c_{2}-$ цикл такой, что

$$
c_{-} b_{-}^{\alpha}=-b_{-}^{\alpha} c_{-}, \quad \text { где } \quad b_{-}^{\alpha}=\frac{\sqrt{p} b_{1}^{\alpha}-\sqrt{q} b_{2}^{\alpha}}{\sqrt{p+q}} .
$$

Запишем теперь уравнение для $\operatorname{str}(g)$ в виде $\operatorname{str}(g)=-\operatorname{str}\left(B^{1} g\right)$ или

$$
\begin{aligned}
\operatorname{str}(g)= & -\operatorname{str}\left(\left(\left[b_{1}^{0}, b_{1}^{1}\right]-1\right) g\right)= \\
= & -\operatorname{str}\left(\left(\left[b_{1}^{0}, b_{1}^{1}\right]-1-\frac{1}{p} \sum_{i=1}^{p} \sum_{j=p+1}^{p+q}\left(\nu_{0} K_{i j}+\nu_{2} K_{i j} R_{i} R_{j}\right)\right) g\right)- \\
& -\operatorname{str}\left(\frac{1}{p} \sum_{i=1}^{p} \sum_{j=p+1}^{p+q}\left(\nu_{0} K_{i j}+\nu_{2} K_{i j} R_{i} R_{j}\right) g\right) .
\end{aligned}
$$


Введем обозначение

$$
h=\left(\left(\left[b_{1}^{0}, b_{2}^{1}\right]-1-\frac{1}{p} \sum_{i=1}^{p} \sum_{j=p+1}^{p+q}\left(\nu_{0} K_{i j}+\nu_{2} K_{i j} R_{i} R_{j}\right)\right) g\right), \quad h \in \mathfrak{G},
$$

и заметим, что $E(h)=e-1$ и $h=\sum_{t} \gamma_{t} g_{t}$ с некоторыми $g_{t} \in G$ и константами $\gamma_{t}$ такими, что все элементы $g_{t}$ содержат цикл $c_{2}$ в их разложении (16). Таким образом, вследствие предположения индукции справедливо следуюшее тождество:

$$
\operatorname{str}(h)=-\operatorname{str}\left(B^{2} h\right)=-\operatorname{str}\left(\left(\left[b_{2}^{0}, b_{2}^{1}\right]-1\right) h\right),
$$

и мы получаем

$$
\begin{aligned}
\operatorname{str}(g)= & \operatorname{str}\left(\left(\left[b_{2}^{0}, b_{2}^{1}\right]-1\right)\left(\left[b_{1}^{0}, b_{1}^{1}\right]-1-\frac{1}{p} \sum_{i=1}^{p} \sum_{j=p+1}^{p+q}\left(\nu_{0} K_{i j}+\nu_{2} K_{i j} R_{i} R_{j}\right)\right) g\right)- \\
& -\operatorname{str}\left(\frac{1}{p} \sum_{i=1}^{p} \sum_{j=p+1}^{p+q}\left(\nu_{0} K_{i j}+\nu_{2} K_{i j} R_{i} R_{j}\right) g\right) .
\end{aligned}
$$

Подстановка $1 \leftrightarrow 2, p \leftrightarrow q$ дает

$$
\begin{aligned}
\operatorname{str}(g)= & \operatorname{str}\left(\left(\left[b_{1}^{0}, b_{1}^{1}\right]-1\right)\left(\left[b_{2}^{0}, b_{2}^{1}\right]-1-\frac{1}{q} \sum_{i=1}^{p} \sum_{j=p+1}^{p+q}\left(\nu_{0} K_{i j}+\nu_{2} K_{i j} R_{i} R_{j}\right)\right) g\right)- \\
& -\operatorname{str}\left(\frac{1}{q} \sum_{i=1}^{p} \sum_{j=p+1}^{p+q}\left(\nu_{0} K_{i j}+\nu_{2} K_{i j} R_{i} R_{j}\right) g\right) .
\end{aligned}
$$

Покажем, что вследствие предположения индукции разность между (П.8) и (П.9) равна нулю.

Эта разность имеет вид

$$
X=\operatorname{str}\left(\left(\frac{\left[b_{2}^{0}, b_{2}^{1}\right]}{p}-\frac{\left[b_{1}^{0}, b_{1}^{1}\right]}{q}\right) \sum_{i=1}^{p} \sum_{j=p+1}^{p+q}\left(\nu_{0} K_{i j}+\nu_{2} K_{i j} R_{i} R_{j}\right) g\right)
$$

т.к. $\operatorname{str}\left(\left[\left[b_{1}^{0}, b_{1}^{1}\right],\left[b_{1}^{0}, b_{1}^{1}\right]\right] g\right)=0$ для всякого суперследа на $\mathfrak{G}$. Теперь мы можем воспользоваться следуюшими тождествами при $1<i \leq p$ и $p+1<j \leq p+q$ :

$$
\begin{aligned}
\left(c_{1}\right)^{-1} K_{i j} c_{1} & =K_{(i-1) j}\left(R_{i-1} R_{j}\right)^{\delta(i-1)}, \\
\left(c_{2}\right)^{-1} K_{i j} c_{2} & =K_{i j-1}\left(R_{i} R_{j-1}\right)^{\delta(j-1)}, \\
\left(c_{1}\right)^{-1} K_{i j} R_{i} R_{j} c_{1} & =K_{(i-1) j}\left(R_{i-1} R_{j}\right)^{\delta(i-1)+1}, \\
\left(c_{2}\right)^{-1} K_{i j} R_{i} R_{j} c_{2} & =K_{i j-1}\left(R_{i} R_{j-1}\right)^{\delta(j-1)+1},
\end{aligned}
$$

чтобы для любого $G$-инвариантного суперследа на $\mathfrak{G}$ представить $X$ в виде $X=$ $X_{0}+X_{2}$, где

$$
\begin{aligned}
& X_{0}=F_{0}\left(\nu_{0}, \nu_{2}\right) \operatorname{str}\left(\left(\frac{\left[b_{2}^{0}, b_{2}^{1}\right]}{p}-\frac{\left[b_{1}^{0}, b_{1}^{1}\right]}{q}\right) K_{1(p+1)} g\right), \\
& X_{2}=F_{2}\left(\nu_{0}, \nu_{2}\right) \operatorname{str}\left(\left(\frac{\left[b_{2}^{0}, b_{2}^{1}\right]}{p}-\frac{\left[b_{1}^{0}, b_{1}^{1}\right]}{q}\right) K_{1(p+1)} R_{1} R_{p+1} g\right),
\end{aligned}
$$

а $F_{0,1}\left(\nu_{0}, \nu_{2}\right)$ - некоторые конкретные функции. Подставляя $b_{2}^{\alpha}=\frac{1}{\sqrt{q}}\left(\sqrt{p+q} B_{+}^{\alpha}-\right.$ $\left.\sqrt{p} b_{1}^{\alpha}\right)$ в $X_{0}$ и $b_{2}^{\alpha}=\frac{1}{\sqrt{q}}\left(\sqrt{p+q} B_{-}^{\alpha}+\sqrt{p} b_{1}^{\alpha}\right)$ в $X_{2}$ и используя предположение индукции и лемму 2 , мы получаем $X_{0}=X_{2}=0$, что завершает доказательство теоремы 2. 


\section{Список литературы}

[1] A. Polychronakos. Phys. Rev. Lett. 1992. V. 69. P. 703.

[2] L. Brink, H. Hansson, M. A. Vasiliev. Phys. Lett. B. 1992. V. 286. P. 109.

[3] F. Calogero. J. Math. Phys. 1969. V. 10. P. 2191, 2197; 1971. V. 12. P. 419.

[4] S. E. Konstein, M. A. Vasiliev. Hep-th/9512038; J. Math. Phys. 1996. V. 37. P. 2872.

[5] M. A. Olshanetsky, A. M. Perelomov. Phys. Rep. 1983. V. 94. P. 313.

[6] L. Brink, M. A. Vasiliev. Mod. Phys. Lett. A. 1993. V. 8. P. 3585.

[7] C. F. Dunkl. Trans. Am. Math. Soc. 1989. V. 311. P. 167.

[8] М. А. Васильев. Письма в ЖЭТФ. 1989. Т. 50. С. 344; M. A. Vasiliev. Int. J. Mod. Phys. A. 1991. V. 6. P. 1115.

Поступила в редакцию 4.IV.1996 г.

\section{SUPERTRACES ON THE ALGEBRAS OF OBSERVABLES OF THE RATIONAL CALOGERO MODELS RELATED TO THE CLASSICAL ROOT SYSTEMS}

We find a complete set of supertraces on the algebra $H_{W(\mathbf{R})}(\nu)$, the algebra of observables of the rational Calogero model with harmonic interaction based on the classical root systems $\mathbf{R}$ of $B_{N}, C_{N}$, and $D_{N}$ types. These results extend the results known for the case $A_{N-1}$. It is shown that $H_{W(\mathbf{R})}(\nu)$ admits $q(\mathbf{R})$ independent supertraces where $q\left(B_{N}\right)=q\left(C_{N}\right)$ is a number of partitions of $N$ into a sum of positive integers and $q\left(D_{N}\right)$ is a number of partitions of $N$ into a sum of positive integers with even number of even integers. 